\title{
Assessment of Different Types of Osteotomies in Rhinoplasty Patients
}

\author{
KARIMA ISMAIL, M.D.; MARIAM ISMAIL, M.D. and AHMED ISMAIL, M.D. \\ The Department of Plastic and Reconstructive Surgery, Faculty of Medicine, Cairo University
}

\begin{abstract}
Introduction: Rhinoplasty is challenging procedure as its one of the aesthetic procedures where the patient and all his acquaintances are constantly seeing and judging the plastic surgeon outcome. Osteotomy is one of the most crucial steps; in order to have good results; it is needed to narrow nasal width. Osteotomy is also one of the difficult steps in rhinoplasty as it is blind and meticulous [1]
\end{abstract}

Material and Methods: Retrospective study from 2010 to 2014 , including 450 patients. Pre-operative data were documented and tabulated. Intraoperative data were documented and tabulated. Post-operative data were documented and tabulated. Follow-up for 6 months and evaluation sheet was filled. 200 patients complied to follow-up, the rest (250 patient) were excluded from the study. Comparative evaluation of the resultswas done and statistically analyzed.

Results: $13 \%$ (26 cases) was total complication rate. Complication varied from;asymmetry: $4.5 \%$, residual hump: $6 \%$, nasal bone collapse: $2 \%$, extensive displacement: $0.5 \%$.

Conclusion: Osteotomy is one of the most crucial steps in rhinoplasty. In this study we did retrospective analysis of 450 rhinoplasty patients and their osteotomy results. Weconcluded that lateral green stick osteotomy with or without median osteotomy is the safest osteotomy.

Key Words: Osteotomies - Rhinoplasty.

\section{INTRODUCTION}

Rhinoplasty is challenging procedure as its one of the aesthetic procedures where the patient and all his acquaintances are constantly seeing and judging the plastic surgeon outcome. Osteotomy is one of the most crucial steps in order to have good results; it is needed to narrow nasal width. Osteotomy is also one of the difficult steps in rhinoplasty as it is blind and meticulous [1].

The type of osteotomies usually can be decided preoperatively; the critical factor is how much movement of the lateral nasal wall is required to narrow the bonybase width. If a great deal of movement is necessary, then one needs complete osteotomies with bony separation. In contrast if one only needs a more limited narrowing, then a greenstick fracture is sufficient. There are several types of osteotomies that can be combined together in order to achieve the desired width reduction however, movement and stability of the lateral walls are critical factors in the decision process [2]. Fig. (1) shows different types of osteotomies.

\section{- Lateral osteotomies:}

A- Low to high osteotomy: Slightly curved osteotome is placed low on the pyriform aperture, then with two-tap sequenced pace its driven up through frontal process of the maxilla to end high at the nasal bone suture line at the level of the medial canthus; followed by a digital compression to produce transverse green stick fracture. Limited movement will be achieved.

B- Low to low osteotomy: Straight osteotome is driven along the base of the frontal process of maxilla, not across it in an ascending fashion; usually this technique is associated with transverse osteotomy to allow complete movement of the lateral nasal bones [3].

- Transverse osteotomies: A vertical stab incision is made just above medial canthus, $2 \mathrm{~mm}$ osteotome is used to completely fracture the lateral wall transversely from just above medial canthus upward. Usually it is followed by low to low osteotomy.

- Medial oblique osteotomies: A curved osteotome is placed at the cephalic end of the open roof and driven downward toward the medial canthus. It is designed to narrow the broad bony dorsum and is coupled with low to low lateral osteotomy [4].

- Double level osteotomies: Consists of an osteotomy along the inferior border of the nasal bone parallel to and combined with a low to low osteotomy. The goal is to reduce the convexity of the lateral wall [5].

- Paramedian osteotomies: Straight osteotomies made $3-5 \mathrm{~mm}$ parallel to the dorsal midline. It is 
used in the broad nose when one does not wish to change dorsal midline [6].

- Microosteotomies: Done with $2 \mathrm{~mm}$ osteotome and are used to correct asymmetries or irregularities intrinsic to the bones [7].

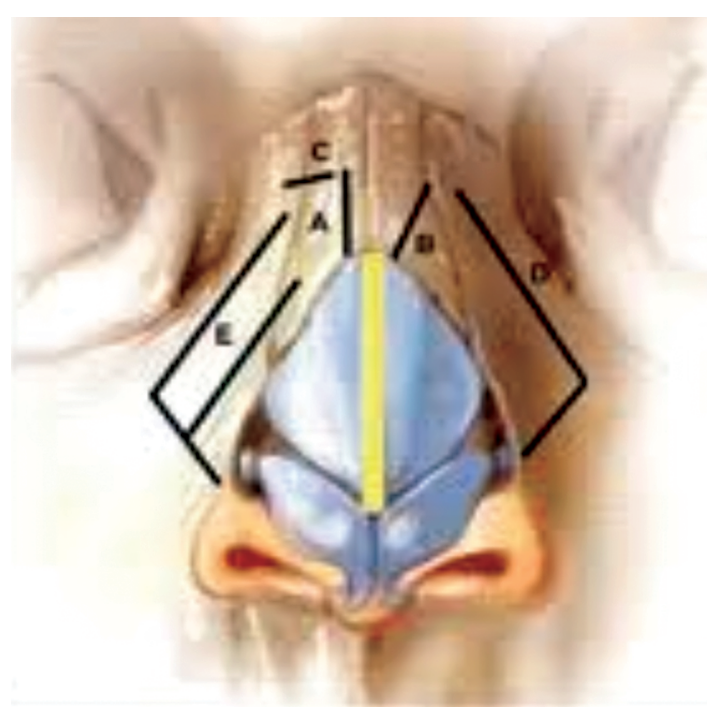

Fig. (1): Types of osteotomies. (A) Paramedian osteotomy. (B) Medial oblique osteotomy. (C) Transverse osteotomy. (D) Low to high osteotomy. (E) Double level osteotomy.

Osteotomy is usually done to narrow bony vault, to close open roof deformity, to create symmetry and to widen bony vault with lateral displacements of nasal bones. Osteotomy is usually critical when patients has short nasal bones, elderly patients with thin, fragile nasal bones and patients with heavy eyeglasses [8].

Complications of osteotomy are either operative trauma or cosmetic complication, Table (1) [9]:

Table (1): Complications of osteotomy.

\begin{tabular}{ll}
\hline Operative trauma & Cosmetic \\
\hline Hemorrhage & Excessive narrowing \\
Edema & Insufficient mobilization \\
Nasal cyst formation & Unstable bony pyramid \\
Anosmia & $\begin{array}{l}\text { Rocker deformity } \\
\text { Arteriovenous fistula }\end{array}$ \\
$\begin{array}{l}\text { Stair step deformity } \\
\text { Epiphora }\end{array}$ & $\begin{array}{l}\text { Infections: Abymmetry } \\
\text { Canicular bleeding } \\
\text { cellulitis. }\end{array}$ \\
Neuromuscular injury & \\
Intracranial injury & \\
\hline
\end{tabular}

\section{MATERIAL AND METHODS}

Retrospective study from 2010 to 2014, including 450 patients. Pre-operative data were documented and tabulated. Intraoperative data were documented and tabulated. Post-operative data were documented and tabulated. Follow-up for 6 months and evaluation sheet by was filled. 200 patients complied to follow-up, the rest (250 patient) were excluded from the study. Comparative evaluation of the results was done and statistically analyzed.

Types of osteotomies used in this study:

- Green stick \& median osteotomy: 62/200 cases, low to low to high lateral osteotomy.

- Greenstick without median osteotomy: 68/200 cases, low to low to high osteotomy.

- Double level osteotomy: 30/200 cases, low to high \& low to low osteotomy.

- Full power osteotomy: 40/200 cases, low to low to high osteotomy.

\section{RESULTS}

$13 \%$ (26 cases) was total complication rate. Complication varied from asymmetry: $4.5 \%$, residual hump: $6 \%$, nasal bone collapse: $2 \%$, extensive displacement: $0.5 \%$.

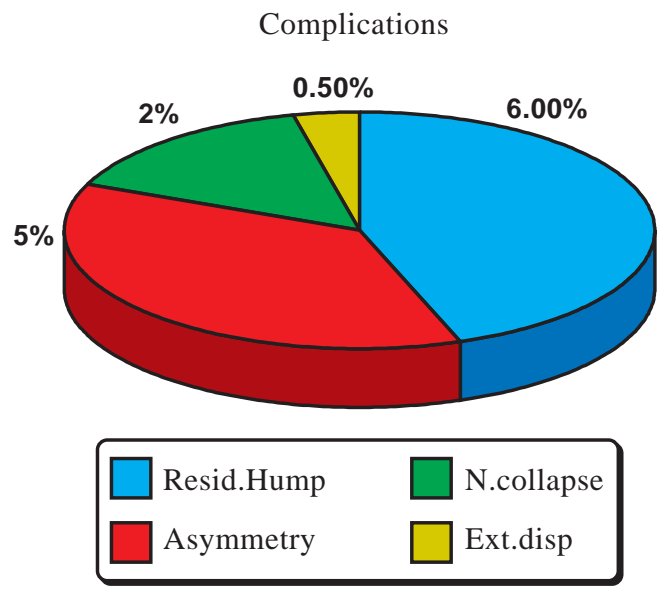

Fig. (2A): Pie chart of complications.

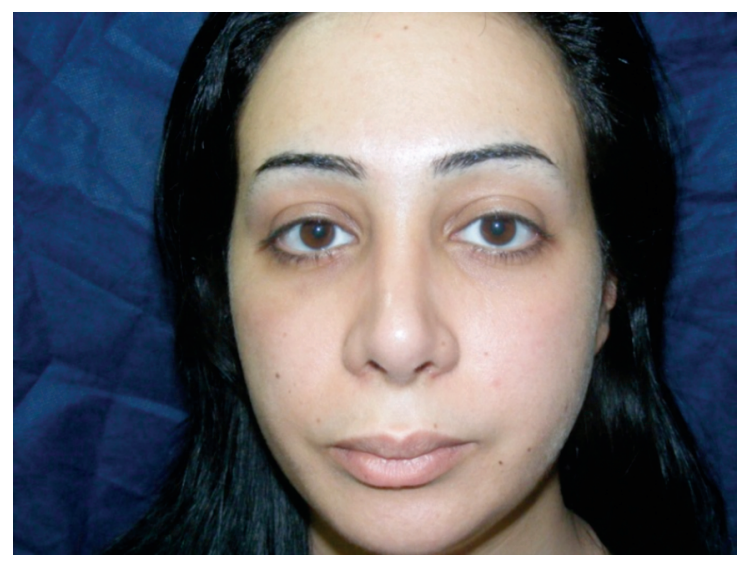

Fig. (2B): Patient showing extensive displacement. 
Complications according to the type of osteotomy:

- Green stick \& median osteotomy: 8 cases [8/62= $12.9 \%, 3$ asymmetry, 4 residual hump, 1 extensive displacement].

- Greenstick without median osteotomy: 5 cases [5/68=7.3\%, 2 asymmetry, 3 residual humps].

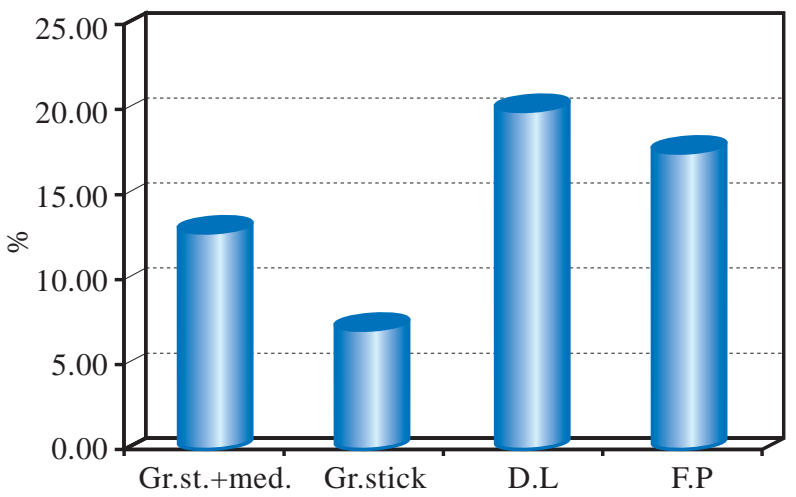

Fig. (3A): Bar chart of complications for each type of osteotomy.

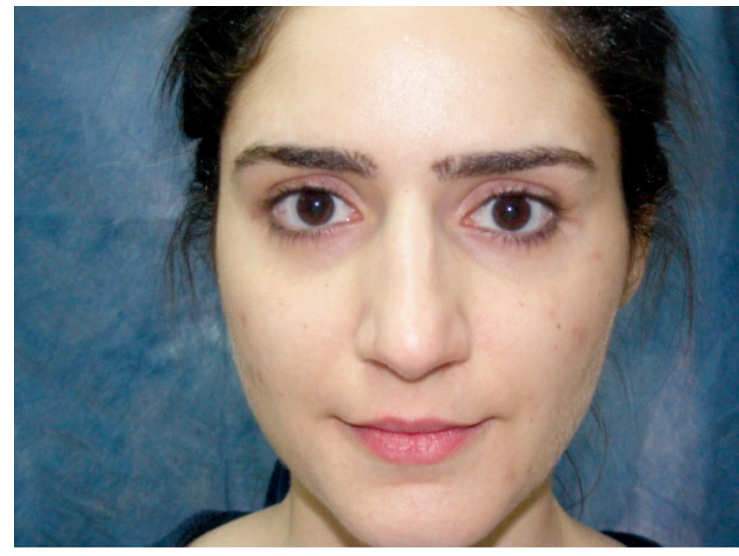

Fig. (4A): Pre-operative rhinoplasty case.

\section{DISCUSSION}

Osteotomy still remains one of the most crucial and challenging steps in rhinoplasty. Therefore, meticulous analysis of osteotomy results in 200 patients was conducted in this study. After tabulating and analyzing the results the following was concluded:

- Safest type of osteotomy is greenstick osteotomy without median osteotomy.

- The next safest osteotomy is green stick with median osteotomy.

- We found that asymmetry, residual hump \& collapse occur more with full power and double level osteotomy.
- Double level osteotomy: 6 cases $[6 / 30=20 \%, 2$ nasal bone collapse, 2 asymmetry, 2 residual humps].

- Full power osteotomy: 7 cases $[7 / 40=17.5 \%, 2$ nasal bone collapse, 2 asymmetry, 3 residual hump].

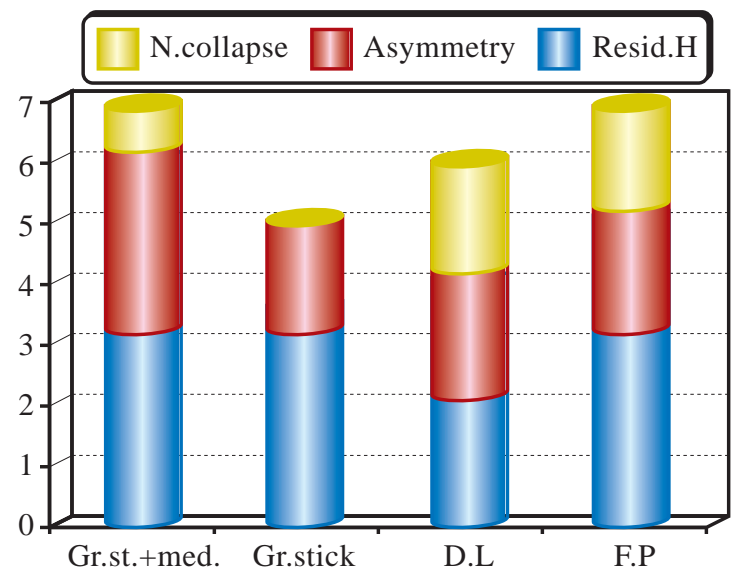

Fig. (3B): Bar chart comparing specific complication to each type of osteotomy.

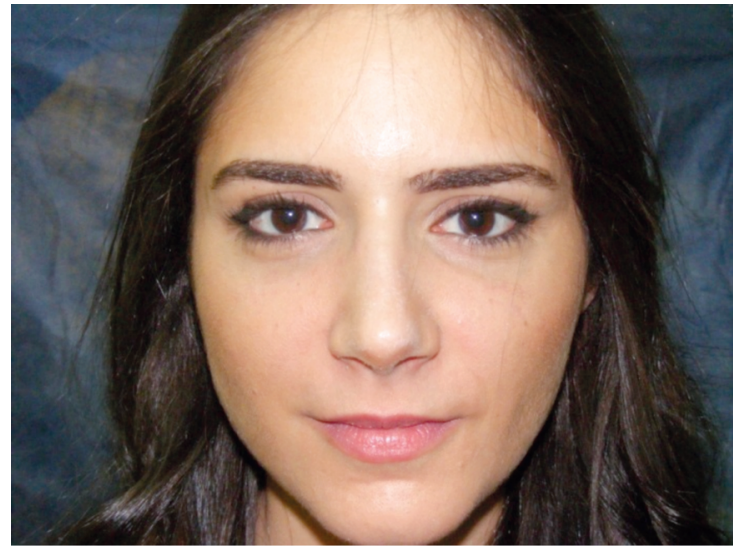

Fig. (4B): Post-operative case after lateral greenstick osteotomy without median osteotomy.

- Low to high osteotomy usually leaves residual step.

- Completion of fracture is done by thumb pressure on small thin bones while thick bones is done by mobilizing the osteotome.

- Our preference is green stick low to low to high osteotomy, with or without median osteotomy.

\section{Conclusion:}

Osteotomy is one of the most crucial steps in rhinoplasty. In this study we did retrospective analysis of 450 rhinoplasty patients and their osteotomy results. We concluded that lateral green stick osteotomy with or without median osteotomy is the safest osteotomy. 


\section{REFERENCES}

1- Gunter J.P., Rohrich R.J. and Adams W.P.: Dallas Rhinoplasty: Nasal Surgery by the Masters, $2^{\text {nded. }}$ St. Louis: Quality Medical Publishing, 2007.

2- Daniel R.: Mastering Rhinoplasty. Berlin: Springer-Verlag, 2010.

3- Gruber R., Chang T., Kahn D., et al.: Broad nasal bone reduction: An algorithm for osteotomies. Plast. Reconstr. Surg., 119: 1044, 2007.

4- Thomas J., Griner N. and Remmler D.: Steps for a safer method of osteotomies in rhinoplasty. Laryngoscope, 97: 746-7, 1987.

5- Parkes M., Kamer F. and Moergan W.: Double lateral osteotomy in rhinoplasty. Arch. Otolaryngol., 103: 344, 1977.

6- Farrior R.: The osteotomy in rhinoplasty. Laryngoscope, 88: 1449, 1978.

7- Tardy M. Jr. and Denneny J.: Micro-osteotomies in rhinoplasty. Facial Plast. Surg., 1: 137, 1984.

8- Rohrich R.J., Krueger J.K., Adams W.P. Jr., et al.: Achieving consistency in the lateral nasal osteotomy during rhinoplasty: An external perforated technique. Plast. Reconstr. Surg., 108: 2122-30; discussion 2131-2, 2001.

9- Beekhius G.J.: Nasal obstruction after rhinoplasty: Etiology, and techniques for correction. Laryngoscope, 86: 540-8, 1976. 